\title{
Vibration of Timoshenko beams using non-classical elasticity theories
}

\author{
J.V. Araújo dos Santos ${ }^{\mathrm{a}, *}$ and J.N. Reddy ${ }^{\mathrm{b}}$ \\ a IDMEC/IST-Instituto Superior Técnico, Av. Rovisco Pais, Lisboa, Portugal \\ ${ }^{\mathrm{b}}$ Mechanical Engineering Department, Texas A\&M University, TAMU, College Station, TX, USA
}

Received 17 August 2009

Revised 7 December 2010

\begin{abstract}
This paper presents a comparison among classical elasticity, nonlocal elasticity, and modified couple stress theories for free vibration analysis of Timoshenko beams. A study of the influence of rotary inertia and nonlocal parameters on fundamental and higher natural frequencies is carried out. The nonlocal natural frequencies are found to be lower than the classical ones, while the natural frequencies estimated by the modified couple stress theory are higher. The modified couple stress theory results depend on the beam cross-sectional size while those of the nonlocal theory do not. Convergence of both non-classical theories to the classical theory is observed as the beam global dimension increases.
\end{abstract}

Keywords: Nonlocal elasticity theory, couple stress theory, Timoshenko beam, rotary inertia, free vibration

\section{Introduction}

The rapid development of nanodevices, such as micro and nano-beams, requires mathematical models that are able to account for interatomic interactions and size effects. Therefore, it is necessary to incorporate into the classical elasticity theory additional material constants. This is accomplished in the nonlocal elasticity theory by assuming that the stress at a certain point is a function of strains at all points of the body [1,2], which leads to a constitutive equation that contains two additional material parameters. Alternative theories that takes into account microstructure-dependent size effects, such as the couple stress theory, have also been developed. The first couple stress theory proposed contains two additional material constants [3-5]. An additional equilibrium equation is included in this theory, which accounts for the equilibrium of moments of forces. Yang et al. [6] developed a modified couple stress theory by assuming that the strain energy density function depends only on the strain and the symmetric part of the curvature tensor, leading to just one additional material constant.

The couple stress theory was applied to model pure bending of circular cylinders [7], while the modified couple stress theory has been recently used in bending and vibration analysis of Euler-Bernoulli [8] and Timoshenko beams [9]. Similar analysis of Euler-Bernoulli [10-12] and Timoshenko beams [13,14] have also been performed using the nonlocal elasticity theory. Analytical solutions for bending, buckling and vibration of Euler-Bernoulli, Timoshenko, Reddy, and Levinson beams were derived by using Hamilton's principle and the nonlocal elasticity theory [15].

In the present study, classical elasticity, nonlocal elasticity, and modified couple stress theories are evaluated in the context of free vibration analysis of Timoshenko beams. The influence of rotary inertia and nonlocal parameters on fundamental and higher natural frequencies is also studied.

\footnotetext{
*Corresponding author. E-mail: viriato@ist.utl.pt.
} 


\section{Theoretical formulation}

The nonlocal theory of elasticity, along with the Timoshenko assumptions [16,17], yield the following equations of motion in terms of the generalized displacements $w$ and $\phi$ of the beam $[15,18]$ :

$$
\begin{aligned}
& G A K_{s}\left(\frac{\partial \phi}{\partial x}+\frac{\partial^{2} w}{\partial x^{2}}\right)=m_{0}\left[\frac{\partial^{2} w}{\partial t^{2}}-\left(e_{0}^{2} a^{2}\right) \frac{\partial^{4} w}{\partial x^{2} \partial t^{2}}\right] \\
& E I \frac{\partial^{2} \phi}{\partial x^{2}}-G A K_{s}\left(\phi+\frac{\partial w}{\partial x}\right)=m_{2}\left[\frac{\partial^{2} \phi}{\partial t^{2}}-\left(e_{0}^{2} a^{2}\right) \frac{\partial^{4} \phi}{\partial x^{2} \partial t^{2}}\right]
\end{aligned}
$$

where $G$ and $E$ are the Young's and shear modulus, respectively, $K_{s}$ is the shear correction factor, and $A, I, m_{0}$ and $m_{2}$ are defined by

$$
A=\int_{A} d A, I=\int_{A} z^{2} d A, m_{0}=\int_{A} \rho d A, m_{2}=\int_{A} \rho z^{2} d A
$$

The parameter that describes the nonlocal effects is $e_{0}^{2} a^{2}$, where $e_{0}$ is a material constant and $a$ is an internal characteristic length [2].

For the modified couple stress theory the equations of motion are [9]:

$$
\begin{aligned}
& G A K_{s}\left(\frac{\partial \phi}{\partial x}+\frac{\partial^{2} w}{\partial x^{2}}\right)-\frac{1}{4} l^{2} G A\left(-\frac{\partial^{3} \phi}{\partial x^{3}}+\frac{\partial^{4} w}{\partial x^{4}}\right)=m_{0} \frac{\partial^{2} w}{\partial t^{2}} \\
& E I \frac{\partial \phi}{\partial x^{2}}-G A K_{s}\left(\phi+\frac{\partial w}{\partial x}\right)-\frac{1}{4} l^{2} G A\left(-\frac{\partial^{2} \phi}{\partial x^{2}}+\frac{\partial^{3} w}{\partial x^{3}}\right)=m_{2} \frac{\partial^{2} \phi}{\partial t^{2}}
\end{aligned}
$$

where $l$ is a material length scale parameter, which measures the effect of the couple stress $[4,8,9]$.

The following expansions of the generalized displacements $w$ and $\phi[15]$

$$
\begin{aligned}
& w(x, t)=\sum_{n=1}^{\infty} W_{n} \sin \frac{n \pi x}{L} e^{i \omega_{n} t}, \\
& \phi(x, t)=\sum_{n=1}^{\infty} \Phi_{n} \cos \frac{n \pi x}{L} e^{i \omega_{n} t}
\end{aligned}
$$

satisfy the simply supported boundary conditions $w=0$ and $M_{x}=0$ for the nonlocal theory of elasticity and $w=0$, $M_{x}=0$, and $Y_{x y}=0$ for the modified couple stress theory, at $x=0, L$, where $M_{x}$ and $Y_{x y}$ are the bending and couple moments, respectively. Nontrivial solutions for $W_{n}$ and $\Phi_{n}$ can be obtained by replacing $w$ and $\phi$ in the equations of motion (1) (or (3)) with the expansions in (4), yielding quadratic equations in $\omega_{n}^{2}$ :

$$
C_{2} \omega_{n}^{4}+C_{1} \omega_{n}^{2}+C_{0}=0
$$

where the constants $C_{1}, C_{2}$, and $C_{3}$ for the nonlocal theory of elasticity are defined by

$$
\begin{aligned}
& C_{2}=\frac{m_{0} m_{2}}{G A K_{s}} \lambda_{n}^{2}, \\
& C_{1}=-\left[m_{0} \Lambda_{n}+m_{2}\left(\frac{n \pi}{L}\right)^{2}\right] \lambda_{n}, \\
& C_{0}=E I\left(\frac{n \pi}{L}\right)^{4},
\end{aligned}
$$

with

$$
\lambda_{n}=1+\left(e_{0}^{2} a^{2}\right)\left(\frac{n \pi}{L}\right)^{2}, \Lambda_{n}=1+\frac{E I}{G A K_{s}}\left(\frac{n \pi}{L}\right)^{2} .
$$


For the modified couple stress theory these constants are given by

$$
\begin{aligned}
& C_{2}=\frac{m_{0} m_{2}}{G A K_{s}}, \\
& C_{1}=-m_{2} \gamma_{n}\left(\frac{n \pi}{L}\right)^{2}-m_{0}\left[\gamma_{n}+\frac{E I}{G A K_{s}}\left(\frac{n \pi}{L}\right)^{2}\right], \\
& C_{0}=\left[E I+\varphi_{n}\right]\left(\frac{n \pi}{L}\right)^{4},
\end{aligned}
$$

with

$$
\gamma_{n}=1+\frac{1}{4} \frac{l^{2}}{K_{s}}\left(\frac{n \pi}{L}\right)^{2}, \varphi_{n}=l^{2} G A+\frac{1}{4} l^{2} \frac{E I}{G K_{s}}\left(\frac{n \pi}{L}\right)^{2} .
$$

If we take into account the rotary inertia $m_{2}, \omega_{n}^{2}$ will be the smallest of the two roots

$$
\omega_{n}^{2}=\frac{-C_{1} \pm \sqrt{C_{1}^{2}-4 C_{0} C_{2}}}{2 C_{2}} .
$$

By neglecting the rotary inertia, i.e. by setting $m_{2}=0$ in Eqs (6) or (8), the solution simplifies to

$$
\omega_{n}^{2}=-\frac{C_{0}}{C_{1}}
$$

yielding

$$
\omega_{n}^{2}=\frac{E I\left(\frac{n \pi}{L}\right)^{4}}{m_{0}\left[1+\frac{E I}{G A K_{s}}\left(\frac{n \pi}{L}\right)^{2}\right]\left[1+\left(e_{0}^{2} a^{2}\right)\left(\frac{n \pi}{L}\right)^{2}\right]}
$$

for the nonlocal elasticity and

$$
\omega_{n}^{2}=\frac{\left[E I+l^{2} G A+\frac{1}{4} l^{2} \frac{E I}{G K_{s}}\left(\frac{n \pi}{L}\right)^{2}\right]\left(\frac{n \pi}{L}\right)^{4}}{m_{0}\left[1+\frac{E I}{G A K_{s}}\left(\frac{n \pi}{L}\right)^{2}+\frac{1}{4} \frac{l^{2}}{K_{s}}\left(\frac{n \pi}{L}\right)^{2}\right]}
$$

for the modified couple stress theory. Note that we recover the classical elasticity natural frequencies, when the rotary is neglected, by setting $e_{0}^{2} a^{2}=0$ in Eq. (12) or $l^{2}=0$ in Eq. (13):

$$
\omega_{n}^{2}=\frac{E I\left(\frac{n \pi}{L}\right)^{4}}{m_{0}\left[1+\frac{E I}{G A K_{s}}\left(\frac{n \pi}{L}\right)^{2}\right]}
$$

Comparing Eqs (12), (13) and (14), we see that the nonlocal theory natural frequencies will be lower than the ones given by the classical elasticity theory, while an opposite trend is revealed in the modified couple stress theory.

\section{Numerical results}

Table 1 contains non-dimensional fundamental frequencies of a beam characterized by the non-dimensional material and geometric parameters [15]: $E=30 \times 10^{6}, \nu=0.3, L=10, b=h=$ varied, $\rho=1, K_{s}=5 / 6$. We see that the effect of the rotary inertia is to decrease the fundamental frequencies, in particular for short beams with $L / h<10$. The effect is more noticeable with the frequencies predicted by the nonlocal elasticity than those corresponding to the modified couple stress theory. Also, while for a given length-to-thickness ratio the variation of the natural frequencies is constant with $e_{0}^{2} a^{2}$, the same is not true for the $l^{2}$ parameter. In the latter case, the influence of the rotary inertia decreases as $l^{2}$ increases. The remaining results presented here are obtained by neglecting the rotary inertia. 
Table 1

Comparison of non-dimensional fundamental frequencies $\left[\bar{\omega}=\omega_{1} L^{2} \sqrt{\frac{m_{0}}{E I}}\right]$ with and without rotary inertia

\begin{tabular}{|c|c|c|c|c|c|c|c|}
\hline \multirow{2}{*}{$L / h$} & \multirow[b]{2}{*}{$\mu$} & \multicolumn{3}{|c|}{ NLET } & \multicolumn{3}{|c|}{ MCST } \\
\hline & & $m_{2}=0$ & $m_{2} \neq 0$ & Diff. [\%] & $m_{2}=0$ & $m_{2} \neq 0$ & Diff. [\%] \\
\hline \multirow[t]{6}{*}{20} & 0 & 9.8381 & 9.8281 & 0.10 & 9.8381 & 9.8281 & 0.10 \\
\hline & 1 & 9.3858 & 9.3763 & 0.10 & 42.8088 & 42.7703 & 0.09 \\
\hline & 2 & 8.9907 & 8.9816 & 0.10 & 58.9232 & 58.8761 & 0.08 \\
\hline & 3 & 8.6416 & 8.6328 & 0.10 & 70.8707 & 70.8203 & 0.07 \\
\hline & 4 & 8.3302 & 8.3218 & 0.10 & 80.5713 & 80.5204 & 0.06 \\
\hline & 5 & 8.0503 & 8.0421 & 0.10 & 88.8001 & 88.7504 & 0.06 \\
\hline \multirow[t]{6}{*}{10} & 0 & 9.7454 & 9.7075 & 0.39 & 9.7454 & 9.7075 & 0.39 \\
\hline & 1 & 9.2973 & 9.2612 & 0.39 & 22.8270 & 22.7480 & 0.35 \\
\hline & 2 & 8.9059 & 8.8713 & 0.39 & 30.3960 & 30.3023 & 0.31 \\
\hline & 3 & 8.5601 & 8.5269 & 0.39 & 36.1297 & 36.0306 & 0.28 \\
\hline & 4 & 8.2517 & 8.2196 & 0.39 & 40.8295 & 40.7299 & 0.24 \\
\hline & 5 & 7.9744 & 7.9434 & 0.39 & 44.8386 & 44.7414 & 0.22 \\
\hline \multirow[t]{6}{*}{5} & 0 & 9.3990 & 9.2740 & 1.35 & 9.3990 & 9.2740 & 1.35 \\
\hline & 1 & 8.9669 & 8.8477 & 1.35 & 13.7057 & 13.5421 & 1.21 \\
\hline & 2 & 8.5894 & 8.4752 & 1.35 & 16.8011 & 16.6213 & 1.08 \\
\hline & 3 & 8.2559 & 8.1461 & 1.35 & 19.2877 & 19.1029 & 0.97 \\
\hline & 4 & 7.9585 & 7.8526 & 1.35 & 21.3870 & 21.2036 & 0.86 \\
\hline & 5 & 7.6910 & 7.5887 & 1.35 & 23.2109 & 23.0332 & 0.77 \\
\hline
\end{tabular}

$\mu=e_{0}^{2} a^{2}$ for NLET and $\mu=l^{2}$ for MCST.

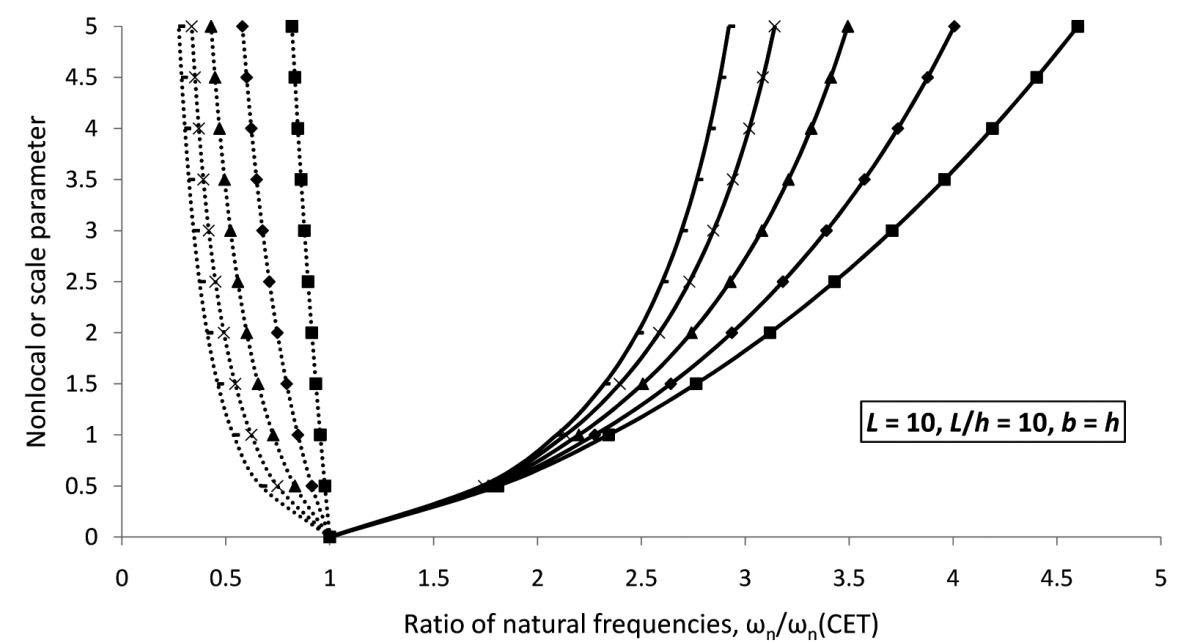

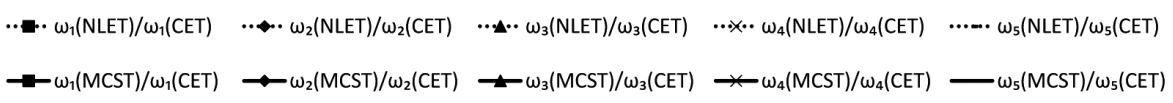

Fig. 1. Dependency of the first five natural frequencies ratios on $e_{0}^{2} a^{2}$ and $l^{2}$.

In order to study the influence of the nonlocal parameter $e_{0}^{2} a^{2}$ and the scale parameter $l^{2}$, we compute the ratio of the natural frequencies $\omega_{n}$ (NLET) estimated by the nonlocal elasticity (or the natural frequencies $\omega_{n}$ (MCST) modified couple stress theory) to those of the classical elasticity theory, $\omega_{n}(\mathrm{CET})$. Both theories converge to the classical local elasticity theory as $e_{0}^{2} a^{2}$ (or $l^{2}$ ) approaches 0 , as shown in Fig. 1. The nonlocal elasticity natural frequencies decrease with an increase in the value of the nonlocal parameter, while for the modified couple stress theory we observe an increase in natural frequencies as the scale parameter is increased. Also, they are much more sensitive to this parameter than to the nonlocal parameter. It was also found that the higher natural frequencies are more affected by the $e_{0}^{2} a^{2}$ parameter, while the parameter $l^{2}$ presents an opposite trend.

In Fig. 2 we observe that with the decrease of width and thickness, there is no variation in the ratio 


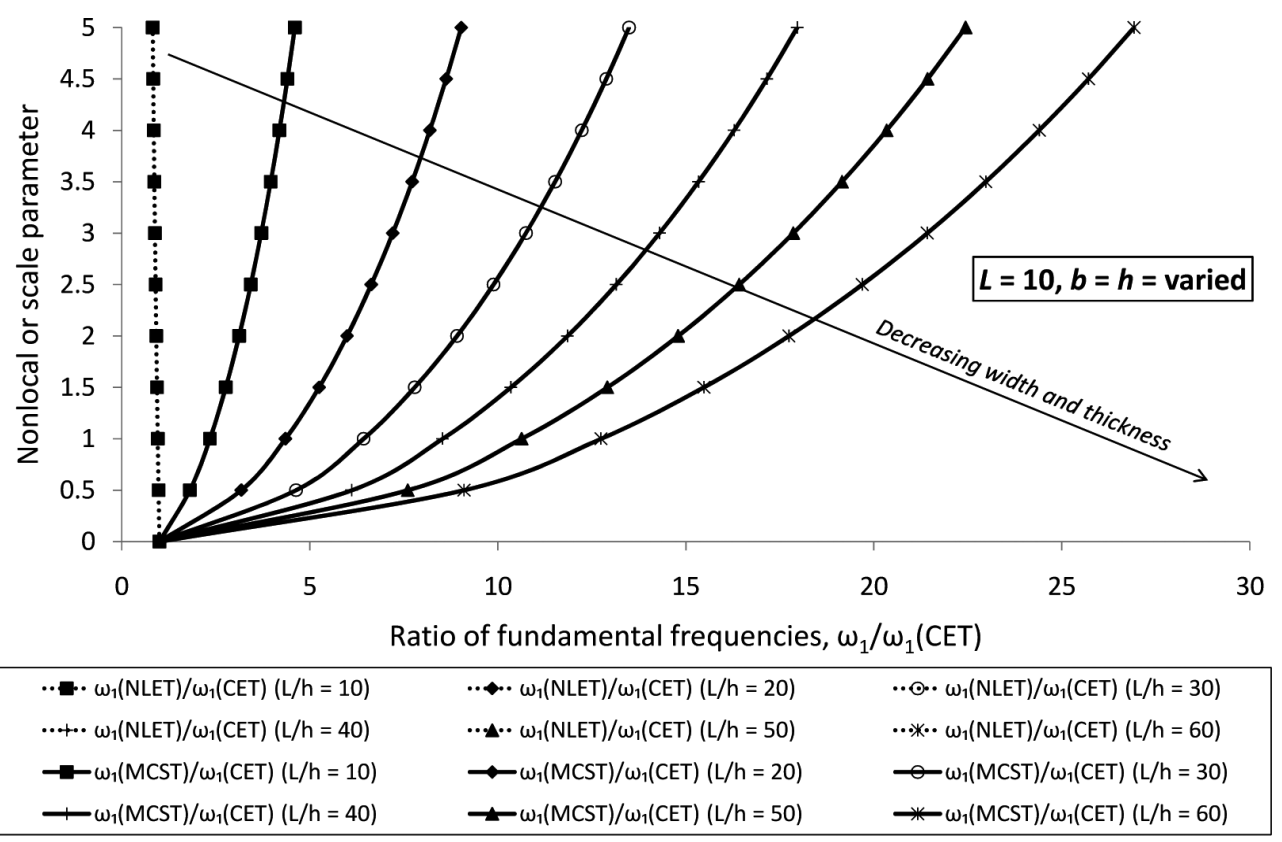

Fig. 2. Dependency of the fundamental frequencies ratios on width and thickness.

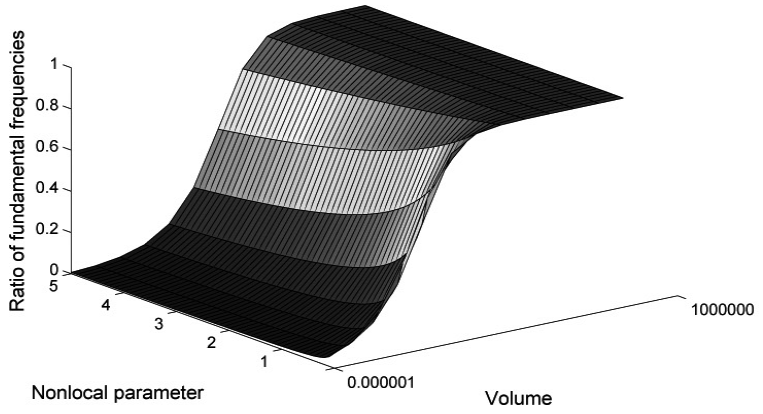

(a)

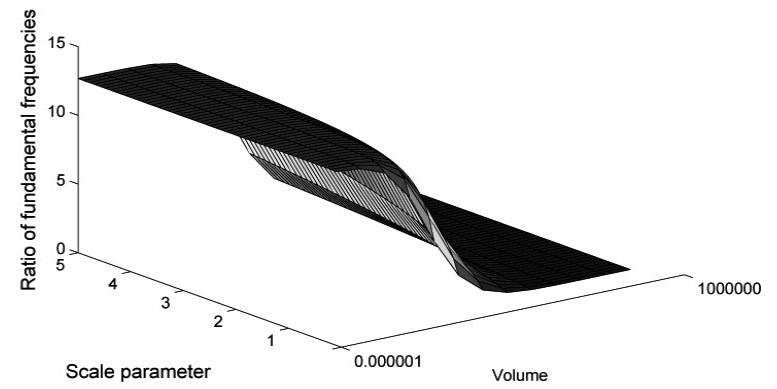

(b)

Fig. 3. Dependency of the fundamental frequencies ratios on the global dimension and a) $e_{0}^{2} a^{2}$, and b) $l^{2}$.

$\omega_{1}(\mathrm{NLET}) / \omega_{1}(\mathrm{CET})$. However, the same does not apply to the ratio $\omega_{1}(\mathrm{MSCT}) / \omega_{1}(\mathrm{CET})$, where an increase of the $l^{2}$ parameter influence is observed as the cross-sectional area decreases. This different trends are due to the introduction of the nonlocal parameters $e_{0}^{2} a^{2}$ through the constitutive equation, while the scale parameter $l^{2}$ appears as a result of the additional presence of the couple stress tensor or its resultant $Y_{x y}$.

Figure 3 shows the ratios of fundamental frequencies as a function of the beam volume, taken as a measure of the global dimension, and the nonlocal or scale parameter. The frequencies are computed by setting $h=b=L / 10$ and varying $L$, in order to keep a constant cross-sectional area-to-length ratio, but increasing volume. We see that both theories converge to the classical elasticity theory for any nonlocal or scale parameter as the beam global dimension increases. This trend is confirmed by evaluating the limit of the frequency ratios as $L \rightarrow \infty$, which takes the value 1 . As the length of the beam decreases, the ratio of the nonlocal natural frequencies to the classical natural frequencies becomes zero. However, the same does not apply to the ratio of the modified couple stress natural frequencies to the classical natural frequencies, which, although independent of $l^{2}$ when $L \rightarrow 0$, is not 1 . This ratio depends on the classical material constants. Note that these trends also are observed for higher natural frequencies. 


\section{Conclusions}

A comparative study of free vibrations of Timoshenko beams based on classical, nonlocal and modified couple stress elasticity theories is carried out. This study shows that the natural frequencies predicted by the nonlocal elasticity theory are smaller than those by the classical elasticity theory and an opposite trend is observed for the natural frequencies predicted by the modified couple stress theory. The nonlocal and couple stress effects become, respectively, more and less pronounced for higher natural frequencies. Although the rotary inertia has a negligible effect, in particular for large length to thickness ratios, its influence is more noticeable in the nonlocal natural frequencies than in the corresponding couple stress ones. Both theories converge to the classical elasticity theory as the beam global dimension increases. Since the nonlocal effect is introduced through the constitutive equation, the nonlocal natural frequencies do not show a cross-sectional size dependency in contrast to the modified couple stress theory. Therefore, the size dependency that was experimentally observed in some materials can not be explained by the nonlocal elasticity theory, which should be discarded in the modeling of some micro- and nano-structures.

\section{Acknowledgments}

The first author gratefully acknowledges the financial support of Fundação para a Ciência e a Tecnologia, through grant SFRH/BPD/48073/2008, while the second author acknowledges the support of the Oscar S. Wyatt Endowed Chair.

\section{References}

[1] A.C. Eringen and D.G.B. Edelen, On nonlocal elasticity, International Journal of Engineering Science 10 (1972), $233-248$.

[2] A.C. Eringen, On differential equations of nonlocal elasticity and solutions of screw dislocations and surface waves, Journal of Applied Physics 54 (1983), 4703-4710.

[3] R.D. Mindlin and H.F. Tiersten, Effects of couple-stresses in linear elasticity, Archive for Rational Mechanics and Analysis 11 (1962), 415-448.

[4] R.D. Mindlin, influence of couple-stresses on stress concentrations, Experimental Mechanics 3 (1962), 1-7.

[5] R.A. Toupin, Theories of elasticity with couple stress, Archive for Rational Mechanics and Analysis 17 (1964), 85-112.

[6] F. Yang, A.C.M. Chong, D.C.C. Lam and P. Tong, Couple stress based strain gradient theory for elasticity, International Journal of Solids and Structures 39 (2002), 2731-2743.

[7] A. Anthoine, Effect of couple-stresses on the elastic bending of beams, International Journal of Solids and Structures 37 (2000), 1003-1018.

[8] S.K. Park and X.-L. Gao, Bernoulli-Euler beam model based on a modified couple stress theory, Journal of Micromechanics and Microengineering 16 (2006), 2355-2359.

[9] H.M. Ma, X.-L. Gao and J.N. Reddy, A microstructure-dependent Timoshenko beam model based on a modified couple stress theory, Journal of the Mechanics and Physics of Solids 56 (2008), 3379-3391.

[10] J. Peddieson, G.R. Buchanan and R.P. McNitt, Application of nonlocal continuum models to nanotechnology, International Journal of Engineering Science 41 (2003), 305-312.

[11] M. Xu, Free transverse vibrations of nano-to-micron scale beams, Proceedings of the Royal Society A 462 (2006), 2977-2995.

[12] Q. Wang and V.K. Varadan, Vibration of carbon nanotubes studied using nonlocal continuum mechanics, Smart Materials and Structures 15 (2006), 659-666.

[13] C.M. Wang, S. Kitipornchai, C.W. Lim and M. Eisenberger, Beam bending solutions based on nonlocal Timoshenko beam theory, Journal of Mechanical Engineering 134 (2008), 475-481.

[14] C.M. Wang, Y.Y. Zhang and X.Q. He, Vibration of nonlocal Timoshenko beams, Nanotechnology 18 (2007), 105401.

[15] J.N. Reddy, Nonlocal theories for bending, buckling and vibration of beams, International Journal of Engineering Science 45 (2007), 288-307.

[16] J.N. Reddy, Energy principles and variational methods in applied mechanics, (Second edition), John Wiley, New York, 2002.

[17] J.N. Reddy, Theory and Analysis of Elastic Plates and Shells, (Second edition), Theory and Analysis of Elastic Plates and Shells, 2007.

[18] J.N. Reddy and S.D. Pang, Nonlocal continuum theories of beams for the analysis of carbon nanotubes, Journal of Applied Physics $\mathbf{1 0 3}$ (2008), 023511. 

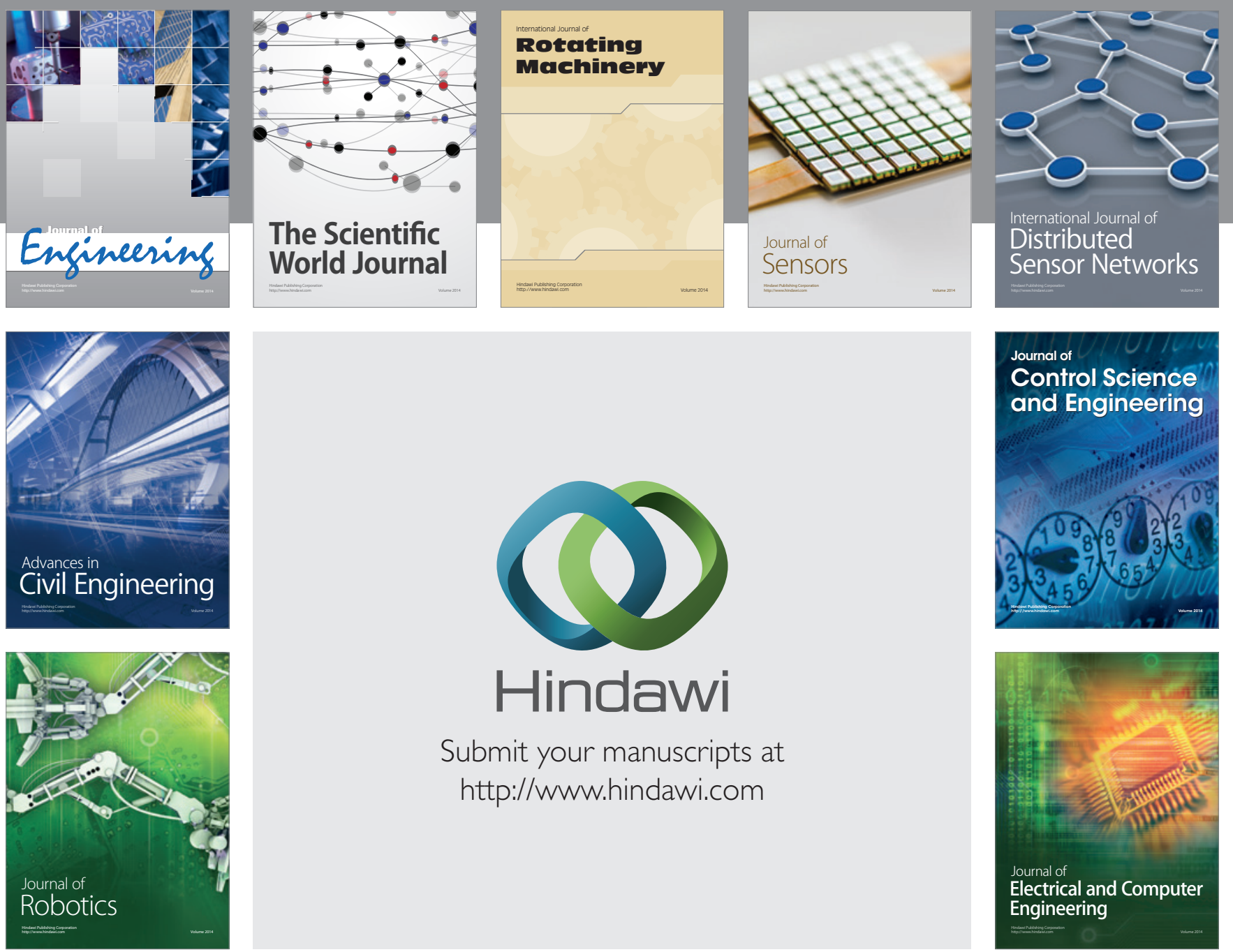

Submit your manuscripts at

http://www.hindawi.com
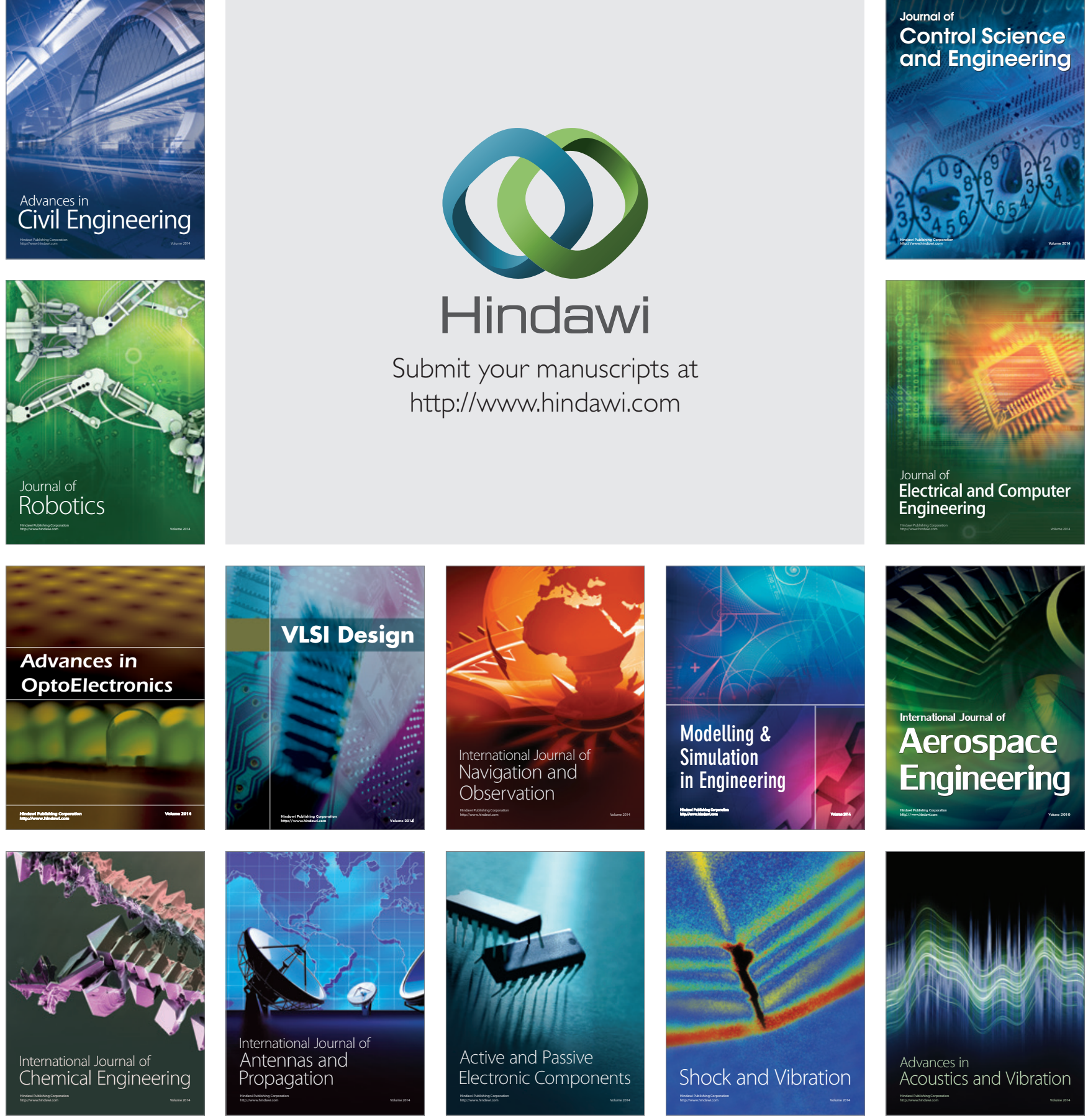